\title{
OPTIMAL INVESTMENT AND BOUNDED RUIN PROBABILITY: CONSTANT PORTFOLIO STRATEGIES AND MEAN-VARIANCE ANALYSIS ${ }^{1}$
}

BY

\author{
Ralf Korn And AnKe Wiese
}

\begin{abstract}
We study the continuous-time portfolio optimization problem of an insurer. The wealth of the insurer is given by a classical risk process plus gains from trading in a risky asset, modelled by a geometric Brownian motion. The insurer is not only interested in maximizing the expected utility of wealth but is also concerned about the ruin probability. We thus investigate the problem of optimizing the expected utility for a bounded ruin probability. The corresponding optimal strategy in various special classes of possible investment strategies will be calculated. For means of comparison we also calculate the related meanvariance optimal strategies.
\end{abstract}

\section{KEYWORDS}

Ruin Process, Optimal Investment, Utility Maximization under Constraints.

\section{INTRODUCTION}

We consider the combined risk and investment process of an insurance company on a finite time horizon $T<\infty$. The insurance risk is described by a classical risk process. The discounted risk process $R$ is thus given by the following model:

$$
R_{t}=x+\int_{0}^{t} c_{s} \mathrm{~d} s-\sum_{i=1}^{N_{t}} X_{i} D_{T_{i}}
$$

where $x$ is the initial reserve of the company, $c_{s}$ is the discounted premium rate at time $s$ per time unit, and $\left(\sum_{i=1}^{N_{t}} X_{i} D_{T_{i}}\right)_{t \in[0, T]}$ denotes the discounted claims process. Here $\left(N_{t}\right)_{t \in[0, T]}$ is a counting process, and $\left(X_{i}\right)_{i \in \mathbb{N}}$ is a sequence of i.i.d.

1 AMS 2000 subject classifications. 60G35, 90A09, 90A43. 
random variables. The variable $X_{i}$ represents the size of the $i$-th claim. The discount factor to time $T_{i}$ of the $i$-th claim is denoted by $D_{T_{i}}$, which is assumed to be strictly positive. See $\mathrm{Ng}$ et al. (2004) for a similar model.

Part of the initial reserve is used for investment in a stock index. By discounting we can and will assume that the price of the riskless asset is constant and equal to one. The discounted stock index is modelled by a geometric Wiener process

$$
\frac{d S_{t}}{S_{t}}=b d t+\sigma d W_{t}
$$

where $b \in \mathbb{R}$ and $\sigma>0$ are constants. Correlation between stock market and bond market is modelled by assuming that the Wiener process $W$ driving the stock market is of the form

$$
W=\rho W^{1}+\bar{\rho} W^{2}
$$

for a constant $\rho \in(-1,+1)$, where $\bar{\rho}=\sqrt{1-\bar{\rho}^{2}}$ and where $\left(W^{1}, W^{2}\right)$ is a standard two-dimensional Wiener process, and $W^{2}$ is independent of the risk process $R$ and of $D$. We do no specify the model of the interest rate process other than through the correlation with the stock market; interest rates are generated by $W^{1}$ and possibly other random sources that are independent of $W^{2}$.

The insurer uses part of the initial reserve, say $z_{0}$, for investment in a stock index. If the insurer follows the investment strategy $\Pi$ in the risky asset, the capital gains from this investment are given by

$$
Z_{t}=z_{0}+\int_{0}^{t} \Pi_{u} d S_{u}=z_{0}+\int_{0}^{t} \Pi_{u}\left\{b S_{u} d u+\sigma S_{u} d W_{u}\right\}
$$

The total wealth of the insurer at time $t \in[0, T]$ discounted to time 0 is then given by

$$
\begin{aligned}
Y(\Pi, t) & =R_{t}-z_{0}+Z_{t} \\
& =x-z_{0}+\int_{0}^{t} c_{s} \mathrm{~d} s+Z_{t}-\sum_{i=1}^{N_{t}} X_{i} D_{T_{i}} .
\end{aligned}
$$

We assume that the insurer has the utility function $U$. The insurer wants to maximize the expected utility of wealth at the end of the time horizon, for instance at the end of the financial year. During this time period, the insurer can borrow unlimited funds at the risk-free rate to pay the claims. However, at the end of the time period, the insurer will have to publish the company report and is also concerned with the ruin probability.

To take into account the ruin probability, we will study the following optimization problem: 
for a given confidence level $\alpha$,

$$
\max _{\Pi \in \Theta} \mathrm{E}[U\{Y(\Pi, T)\}],
$$

subject to $P\{Y(\Pi, T) \leq 0\} \leq \alpha$.

Note that due to the fact the discount factors are strictly positive, the constraint in (5) is unaffected by discounting.

Optimization problems with constraints for investments in a setting without claims are considered for instance by Basak \& Shapiro (2001), Emmer et al. (2001), and Gandy (2005) in the case when the risky asset is driven by a geometric Wiener process, and for exponential Lévy processes by Emmer \& Klüppelberg (2004) using a Gaussian approximation.

The situation is different when a claims process in which trading is not possible is introduced. The question to find the optimal investment that minimizes the ruin probability or that maximizes expected utility in different settings has been studied among others by Browne (1995), Hipp \& Plum (2000, 2003), and Gaier et al. (2003). Estimates for the ruin probability in different settings are derived in Paulsen (2002), Schmidli (2005), and for a diffusion approximation in Browne (1995). See also Paulsen (1998) and Yuen et al. (2004) for ruin probabilities in models with stochastic investment return.

As the task of maximizing over all possible investment strategies (i.e. squareintegrable, predictable processes) is a very complicated task in the presence of the constraint on the ruin probability, we will in the following consider various specialized subclasses of investment strategies. Therefore, $\Theta$ denotes a suitable such class of investment strategies.

In this paper, we will restrict $\Theta$ to the following three classes which are popular in practical applications:

- The class $\Theta_{p}$ of investment strategies, where a constant proportion of capital gains is continuously invested in the risky asset. Here, the term proportion corresponds solely to the wealth process $Z$ that starts with an initial wealth of $z_{0} \leq x$ and then is a consequence of the investment activities, formerly separated from the insurance cash flows.

- The class $\Theta_{b}$ of buy-and-hold strategies, where the initial number of shares of the risky asset bought is kept constant until the time horizon.

- The class $\Theta_{a}$ of investment strategies, where for all times $t \in[0, T]$ the investment in the risky asset is kept to a constant amount.

These classes of investment strategies are shown to be optimal for different investment problems. Constant proportion strategies are ubiquitous in portfolio optimization, see Merton (1971) and numerous variations of the Merton problem. Constant amount strategies in particular for investment problems with constraints are shown to be optimal in different settings and under different optimality criteria by Gaier et al. (2003) or Browne (1995, 1997). Buy-and-hold strategies are 
commonly employed in the context of portfolio performance evaluation where they are compared with other investment strategies, see Perold \& Sharpe (1988), Ferson \& Schadt (1996) or Loviscek \& Jordan (2000). See also Hipp \& Plum $(2003)$ or Browne $(1995,1997)$ for related problems for which the optimal strategy is not constant.

As the total wealth is assumed to be collected only at $T$ the end of the investment/business period, it seems reasonable to separate the initial wealth at time $t=0$ into a part $z_{0}$ usable for investment and the remaining part $x-z_{0} \geq 0$ that can (or has to) be used as a safety cushion for the insurance claims. This separation is assumed to be given and can indeed have legal motivation such as the rules of Solvency II.

We will see that in all situations that are of practical interest, the solution to the constrained optimization problem (5) for either class $\Theta$ of strategies considered above exists and that it is finite. For many interesting utility functions, the solution is given by the largest value for which the constraint is fulfilled. This is due to the fact that for these classes of investment strategies the ruin probability of the combined investment-ruin process is continuous as a function of investment, while at the same time its asymptotic behaviour is known. Ruin constraints $\alpha$ can thus be chosen suitably so that optimization is performed over a compact and non-empty set. Note in particular that these arguments do not rely on the assumption that the insurance risk is described by a classical ruin process.

In a section containing numerical examples, we investigate some of the explicit solutions of the optimal portfolio problems in the different classes of investment strategies. We compare the case when there are many small claims with the case of few large claims. We also compare the characteristic behaviour of the solution to our optimization problems with that of corresponding mean-variance problems. We will see that in the situation of rare large claims the potential of the risky investment to generate a lot of capital gains before the first big claim occurs is used to satisfy the ruin probability constraint, and consequently the optimal investment in the stock market is considerably larger compared to the one in the situation of frequent small claims.

We will also investigate the related problem, when the ruin probability is monitored continuously in the following sense:

for a given confidence level $\alpha$,

$$
\begin{gathered}
\max _{\Pi \in \Theta} \mathrm{E}[U\{Y(\Pi, T)\}], \\
\text { subject to } \sup _{t \in[0, T]} P\{Y(\Pi, t) \leq 0\} \leq \alpha .
\end{gathered}
$$

This problem can be treated by extending the arguments for the optimization problem (5), and we will see that similar results hold. Since

$$
\sup _{t \in[0, T]} P\{Y(\Pi, t) \leq 0\} \leq P\left\{\inf _{t \in[0, T]} Y(\Pi, t) \leq 0\right\},
$$


a solution to (6) does not necessarily guarantee that the probability of ruin before $T$,

$$
P\left\{\inf _{t \in[0, T]} Y(\Pi, t) \leq 0\right\} \leq \alpha,
$$

is also bounded by $\alpha$. However, the condition in (6) guarantees that the probability of ruin is controlled pointwise in $t$ over the entire time period $[0, T]$. Dealing with the condition (8) in the presence of a non-diffusion risk process seems in general to be impossible.

The paper is outlined as follows. In Section 2, we present the solution to optimization problem (5). For comparison, we study the corresponding mean-variance problems in Section 3. The optimization problem (6) is solved in Section 4 for all classes of investment strategies $\Theta_{p}, \Theta_{b}$, and $\Theta_{a}$. Lastly, Section 5 contains our numerical examples.

\section{Optimal Constant Portfolios}

Every investment strategy $\Pi$ in the class $\Theta_{p}$ is given by

$$
\Pi_{t} S_{t}=\pi Z_{t}
$$

for a constant $\pi \in \mathbb{R}$ where $Z$ is the wealth process of our investment capital. The process $Z=Z^{\pi}$ for such an investment is thus given by

$$
\begin{aligned}
Z_{t}^{\pi} & =z_{0}+\int_{0}^{t} \pi Z_{u}^{\pi} \frac{d S_{u}}{S_{u}} \\
& =z_{0} \exp \left[\left(\pi b-\frac{1}{2} \pi^{2} \sigma^{2}\right) t+\pi \sigma W_{t}\right] .
\end{aligned}
$$

In the class $\Theta_{b}$ of buy-and-hold strategies, the wealth $Z_{t}=Z_{t}^{z}$ at time $t$ corresponding to a buy-and-hold strategy with initial investment of $z$ units of money in the risky asset is given by

$$
Z_{t}^{z}=z e^{\left(b-\frac{1}{2} \sigma^{2}\right) t+\sigma W_{t}} .
$$

After the choice of $z$ we are thus again in the setting of the class $\Theta_{p}$, as the evolution of the wealth process is the same as one with a special constant portfolio process, namely

$$
\pi=1 \text {. }
$$

In the class $\Theta_{a}$, every investment strategy $\Pi$ is of the form

$$
\Pi_{t}=\frac{\pi}{S_{t}},
$$


where $\pi$ is a constant. Consequently, the capital gains process from this investment $Z=Z^{\pi}$ is given at time $t$ by

$$
\begin{aligned}
Z_{t}^{\pi} & =z_{0}+\int_{0}^{t} \frac{\pi}{S_{u}} d S_{u}=z_{0}+\pi b t+\pi \sigma W_{t} \\
& \sim N\left(z_{0}+\pi b t, \pi^{2} \sigma^{2} t\right) .
\end{aligned}
$$

For notational convenience, we will identify an investment strategy $\Pi$ with its corresponding fraction or its corresponding amount, $\pi$, respectively, and for a buy-and-hold strategy with its initial capital $z$. We will write $Y(\pi, t)=Y(\Pi, t)$ and $Y(z, t)$, respectively, for short.

It will further prove to be useful to introduce the following notation:

$$
\alpha_{0}(d):=P\left\{R_{T} \leq d\right\}, \quad \alpha_{\pi}(d):=P\{Y(\Pi, T) \leq d\}
$$

for all $d \in \mathbb{R}$ and a fixed value of $z_{0}$.

Lemma 2.1. The ruin probability is continuous as a function of the investment strategy and has the following asymptotic behaviour for all $d \in \mathbb{R}$ :

a) In the class $\Theta_{p}$ of constant proportion strategies and for a given initial capital $z_{0} \in[0, x]$, it is

$$
\lim _{\pi \rightarrow \pm \infty} \alpha_{\pi}(d)=\alpha_{0}\left(d+z_{0}\right) .
$$

b) In the class $\Theta_{b}$ of buy-and-hold strategies, it is

$$
\lim _{z \rightarrow \pm \infty} P\{Y(z, T) \leq d\}=\Phi\left(\mp \frac{b-\frac{1}{2} \sigma^{2}}{\sigma} \sqrt{T}\right) .
$$

c) In the class $\Theta_{a}$ of constant amount strategies, and for a given initial capital $z_{0} \in[0, x]$, we have

$$
\lim _{\pi \rightarrow \pm \infty} P\{Y(\pi, T) \leq d\}=\Phi\left(\mp \frac{b}{\sigma} \sqrt{T}\right) .
$$

Here $\Phi$ denotes the distribution function of the standard Normal distribution.

Proof: a) Note that $Y(\pi, T)$ given $\left(R_{T}, W_{T}^{1}\right)$ has a lognormal distribution on the set $\left\{R_{T} \leq d+z_{0}\right\}$, and thus

$$
\begin{array}{r}
P\left\{x-z_{0}+\int_{0}^{T} c_{s} \mathrm{~d} s+Z_{T}^{\pi}-\sum_{i=1}^{k} X_{i} D_{T_{i}} \leq d \mid\left(R_{T}, W_{T}^{1}\right)\right\} \\
=\Phi\left(\frac{\ln \left(\frac{d+z_{0}-R_{T}}{z_{0}}\right)-\left(\pi b-\frac{1}{2} \pi^{2} \bar{\rho}^{2} \sigma^{2}\right) T-\pi \rho \sigma W_{T}^{1}}{|\pi| \sigma \bar{\rho} \sqrt{T}}\right)
\end{array}
$$


on $\left\{R_{T} \leq d+z_{0}\right\}$ and it is zero otherwise. Continuity in $\pi$ is thus immediate. Since

$$
\begin{aligned}
& \frac{\ln \left(\frac{d+z_{0}-R_{T}}{z_{0}}\right)-\left(\pi b-\frac{1}{2} \pi^{2} \sigma^{2} \bar{\rho}^{2}\right) T-\pi \rho \sigma W_{T}^{1}}{|\pi| \sigma \bar{\rho} \sqrt{T}} \\
& =\frac{\text { const }}{|\pi|}-\operatorname{sign}(\pi) \frac{\left(b-\frac{1}{2} \pi \sigma^{2} \bar{\rho}^{2}\right) T+\rho \sigma W_{T}^{1}}{\sigma \bar{\rho} \sqrt{T}} \rightarrow \infty
\end{aligned}
$$

for $\pi \rightarrow \pm \infty$, it follows that

$$
\lim _{\pi \rightarrow \pm \infty} P\{Y(\pi, T) \leq d\}=P\left\{R_{T} \leq d+z_{0}\right\}=\alpha_{0}\left(d+z_{0}\right) .
$$

b) Equation (18) with $\pi=1$ and the corresponding equation for negative $z_{0}$ implies that for any $d \in \mathbb{R}$ the map

$$
z \mapsto P\left\{R_{T}-z+Z_{T}^{z} \leq d\right\}
$$

is continuous with

$$
\begin{aligned}
\lim _{z \rightarrow \pm \infty} P\left\{R_{T}-z+Z_{T}^{z} \leq d\right\} & =E\left[\Phi\left(\mp \frac{\left(b-\frac{1}{2} \sigma^{2}\right) T+\rho \sigma W_{T}^{1}}{\sigma \bar{\rho} \sqrt{T}}\right)\right] \\
& =\Phi\left(\mp \frac{b-\frac{1}{2} \sigma^{2}}{\sigma} \sqrt{T}\right)
\end{aligned}
$$

by Lemma IV.2 in Korn \& Korn (1999).

c) The total wealth of the insurer given $\left(R_{T}, W_{T}^{1}\right)$ at time $T$ has a normal distribution:

$$
P\left\{R_{T}-z_{0}+Z_{T}^{\pi} \leq d \mid\left(R_{T}, W_{T}^{1}\right)\right\}=\Phi\left(\frac{d-R_{T}-\pi\left(b T+\sigma \rho W_{T}^{1}\right)}{\sigma|\pi| \bar{\rho} \sqrt{T}}\right) .
$$

Consequently, the distribution function of the total wealth as a function in $\pi$ has the following asymptotic behaviour

$$
\begin{aligned}
\lim _{\pi \rightarrow \pm \infty} P\left\{R_{T}-z_{0}+Z^{\pi} \leq d\right\} & =E\left[\Phi\left(\mp \frac{b T+\sigma \rho W_{T}^{1}}{\sigma \bar{\rho} \sqrt{T}}\right)\right] \\
& =\Phi\left(\mp \frac{b}{\sigma} \sqrt{T}\right)
\end{aligned}
$$


by Lemma IV.2 in Korn \& Korn (1999), and

$$
\lim _{\pi \rightarrow 0} P\left\{R_{T}-z_{0}+Z^{\pi} \leq d\right\}=P\left\{R_{T} \leq d\right\} .
$$

Continuity is now immediate.

We define the following upper bounds for the ruin probability $\alpha$ for each class of admissible strategies:

$$
\begin{aligned}
& B_{p}:=P\left\{R_{T} \leq z_{0}\right\} . \\
& B_{b}:=\min \left\{\Phi\left(-\frac{b-\frac{1}{2} \sigma^{2}}{\sigma} \sqrt{T}\right), \Phi\left(\frac{b-\frac{1}{2} \sigma^{2}}{\sigma} \sqrt{T}\right)\right\} \\
& B_{a}:=\min \left\{\Phi\left(-\frac{b}{\sigma} \sqrt{T}\right), \Phi\left(\frac{b}{\sigma} \sqrt{T}\right)\right\} .
\end{aligned}
$$

Theorem 2.2. Let $i \in\{p, b, a\}$. Assume that for a given confidence level

$$
\alpha<B_{i},
$$

there exists a strategy $\pi \in \Theta_{i}$ such that

$$
P\{Y(\pi, T) \leq 0\} \leq \alpha .
$$

Let $U$ denote the utility function of the insurer. If the expected utility of terminal wealth $E[U\{Y(\pi, T)\}]$ is continuous in $\pi \in \mathbb{R}$ or if $E[U\{Y(\pi, T)\}]$ is monotone increasing in $\pi$, then there exists a finite solution $\pi^{*}$ of optimization problem (6) in the class of investment strategies $\Theta_{i}$. In the latter case, $\pi^{*}$ is given by the largest value for which the constraint is fulfilled.

Proof: By Lemma 2.1, the ruin probability

$$
P\{Y(\pi, T) \leq 0\}
$$

is continuous in $\pi \in \mathbb{R}$, and for $\alpha<B_{i}, i \in\{p, b, a\}$, the set

$$
\{\pi \in \mathbb{R}: P\{Y(\pi, T) \leq 0\} \leq \alpha\}
$$

is a compact subset of $\mathbb{R}$, which is non-empty by assumption. Thus, if $E[U\{Y(\pi, T)\}]$ is continuous or if $E[U\{Y(\pi, T)\}]$ is monotone increasing in $\pi$, then an optimal 
solution $\pi^{*}$ to optimization problem (5) exists and is finite. If $E[U\{Y(\pi, T)\}]$ is monotone increasing, then $\pi^{*}$ is given by

$$
\pi^{*}=\sup \{\pi \in \Theta: P\{Y(\pi, T) \leq 0\} \leq \alpha\} .
$$

Corollary 2.3. Assume that the utility function $U$ is such that $E[U\{Y(\pi, T)\}]$ is continuous in $\pi \in \mathbb{R}$ or that $E[U\{Y(\pi, T)\}]$ is monotone increasing in $\pi$.

a) For ruin probabilities

$$
\alpha \in\left[P\left\{R_{T} \leq 0\right\}, P\left\{R_{T} \leq z_{0}\right\}\right)
$$

there exists a finite solution $\pi^{*} \in \Theta_{p}$ of optimization problem (5).

b) If $P\left\{R_{T} \leq 0\right\}<B_{b}$, then for

$$
\alpha \in\left[P\left\{R_{T} \leq 0\right\}, B_{b}\right)
$$

there exists a finite solution $\pi^{*} \in \Theta_{b}$ of optimization problem (5).

c) If $P\left\{R_{T} \leq 0\right\}<B_{a}$, then for

$$
\alpha \in\left[P\left\{R_{T} \leq 0\right\}, B_{a}\right)
$$

there exists a finite solution $\pi^{*} \in \Theta_{a}$ of optimization problem (5).

Proof: Lemma 2.1 implies that for each of these choices of $\alpha$, there exists an admissible finite strategy $\pi \in \mathbb{R}$ such that

$$
P\{Y(\pi, T) \leq 0\} \leq \alpha .
$$

Existence of an optimal strategy now follows from Theorem 2.2.

Note that in the situation of Corollary 2.3, if for $i \in\{p, b, a\}$ the ruin probability $\alpha$ is chosen to be larger than $B_{i}$ and if $E[U\{Y(\pi, T)\}]$ is monotone increasing, then due to Lemma $2.1 \pi^{*}=\infty$ is optimal.

Remark 2.4. a) Consider in particular the risk-neutral utility function

$$
U(x)=x .
$$

Then the expected utility, $E[U\{Y(\pi, T)\}]$, is monotone increasing and continuous in $\pi$ for each class $\Theta_{p}, \Theta_{b}$, and $\Theta_{a}$. Thus, to solve the corresponding portfolio problem of maximizing the expected final wealth while keeping the ruin probability below a given constant $\alpha$ we only have to look for the biggest 
portfolio process $\pi$ with a ruin probability not exceeding it. This, however, can be a demanding task depending on the distribution of the total insurance claims. We therefore refer to our numerical examples in the last section of the paper.

b) Note that for short time horizons $T$ and for realistic values of $b$ and $\sigma$, the inequalities $\alpha<B_{a}$ and $\alpha<B_{b}$ are always fulfilled for ruin probabilities $\alpha$ that are of practical interest. On the other hand we note that the right-hand sides of inequalities (26) and (27) are decreasing in $T$. In particular, if we consider the utility function $U(x)=x$, then for any given ruin probability $\alpha$, and a sufficiently long time horizon $T$, in the class $\Theta_{a}$ the optimal investment is given by $\pi^{*}=\infty$ if the stock price drift $b>0$, and by $\pi^{*}=-\infty$ if $b<0$, and in the class $\Theta_{b}$ the optimal investment is given by $z^{*}=\infty$ if $b-\frac{1}{2} \sigma^{2}>0$, and by $z^{*}=-\infty$ if $b-\frac{1}{2} \sigma^{2}<0$.

c) Assume $\rho=0$. A further suitable choice for utility functions for the class of constant amount investment strategies $\Theta_{a}$, other than the risk-neutral utility function discussed in a) above, is the exponential utility function

$$
U(x)=1-\exp (-\gamma x) \text { for some } \gamma>0
$$

as we then have:

$$
\begin{gathered}
E(1-\exp (-\gamma Y(\Pi, t))) \\
=1-\exp \left(-\gamma \pi b t-\frac{1}{2} \gamma^{2} \pi^{2} \sigma^{2} t\right) E\left(\exp \left(-\gamma R_{t}\right)\right) .
\end{gathered}
$$

In this case, similar to the linear utility function considered above, we have explicit functional dependence of $\pi$ and only the non-specified expectations of the risk process are unknown. Note that while for the choice of the linear utility function, the expected utility is strictly increasing in $\pi$ given a positive drift rate of the stock price, for the choice of the exponential utility this is only ensured for

$$
\pi \in\left(-\infty, \frac{b}{\gamma \sigma^{2}}\right]
$$

while on the remaining part of the real line it strictly decreases as a function of $\pi$.

\section{Mean-Variance Analysis}

Related problems that can be solved explicitly are the mean-variance optimization problems with regard to the classes of investment strategies we considered in the preceding sections. Note therefore that we have

$$
\begin{aligned}
\operatorname{Var}(Y(\pi, T))= & \operatorname{Var}\left(R_{T}\right)+z_{0}^{2} e^{2 b \pi T}\left(e^{\pi^{2} \sigma^{2} T}-1\right) \\
& +2 z_{0} e^{\pi b T-\frac{1}{2} \pi^{2} \sigma^{2} T} \operatorname{Cov}\left(R_{T}, e^{\pi \sigma \rho W_{T}^{1}}\right),
\end{aligned}
$$


for $\pi \in \Theta_{p}$. In particular, for $\pi=1$, we obtain the variance for a buy and hold strategy characterized by the initial amount of $z_{0}$ invested in the risky asset. As for $\Pi \in \Theta_{a}$, we also have

$$
\operatorname{Var}(Y(\pi, T))=\operatorname{Var}\left(R_{T}\right)+\pi^{2} \sigma^{2} T+2 \pi \sigma \rho \operatorname{Cov}\left(R_{T}, W_{T}^{1}\right),
$$

we have explicit solutions for all mean-variance problems of the form

$$
\begin{aligned}
\max _{\Pi \in \Theta} & \mathrm{E}[Y(\pi, T)] \\
& \text { subject to } \operatorname{Var}(Y(\pi, T)) \leq \beta .
\end{aligned}
$$

Theorem 3.1. Assume that we have $b, \rho>0$ and

$$
\beta \geq \operatorname{Var}\left(R_{T}\right) .
$$

Then the solution of the above mean-variance problem is given by

a) the largest solution $\pi^{*}$ of

$$
\begin{aligned}
\beta-\operatorname{Var}\left(R_{T}\right)= & z_{0}^{2} e^{2 b \pi T}\left(e^{\pi^{2} \sigma^{2} T}-1\right) \\
& +2 z_{0} e^{\left(\pi b-\frac{1}{2} \pi^{2} \sigma^{2} \rho^{2}\right) T} \operatorname{Cov}\left(R_{T}, e^{\pi \sigma \rho W_{T}^{1}}\right)
\end{aligned}
$$

in the case of constant proportion portfolio strategies.

b) the initial amount of

$$
\begin{aligned}
z= & +\frac{1}{e^{b T} \sqrt{e^{\sigma^{2} T}-1}} \sqrt{\beta-\operatorname{Var}\left(R_{T}\right)+\frac{e^{-\sigma^{2} \rho^{2} T}}{e^{\sigma^{2} T}-1} \operatorname{Cov}\left(e^{\sigma \rho W_{T}^{1}}, R_{T}\right)^{2}} \\
& -\frac{e^{-\frac{1}{2} \sigma^{2} \rho^{2} T}}{e^{b T} \sqrt{e^{\sigma^{2} T}-1}} \operatorname{Cov}\left(e^{\sigma \rho W_{T}^{1}}, R_{T}\right)
\end{aligned}
$$

in the case of buy and hold strategies.

c) the constant amount

$$
\pi=+\frac{1}{\sigma \sqrt{T}} \sqrt{\beta-\operatorname{Var}\left(R_{T}\right)+\frac{\rho^{2}}{T} \operatorname{Cov}\left(R_{T}, W_{T}^{1}\right)^{2}}-\frac{\rho}{\sqrt{T}} \operatorname{Cov}\left(R_{T}, W_{T}^{1}\right)
$$

Proof: The proof of the theorem follows directly from the explicit form of the variance given above and the fact that the expectation is increasing in $\Pi$ for 
positive stock price drift $b$. Note that the right-hand side in (32) is continuous in $\pi$, equals zero for $\pi=0$, and tends to infinity for $\pi \rightarrow \infty$.

Note in particular that for a given value of $\beta$, the solution to the optimization problem is for all three classes of investment strategies independent of the initial wealth $x$. Of course, as a consequence of this it can also be possible that the optimal stock position has to be partly financed by a credit. This could be avoided by additionally requiring a suitable bound for the stock investment. The obvious consequence of such an additional constraint is that then the optimal investment in the stock is given by the minimum of this bound and the solution in Theorem 3.1.

\section{Optimal InVESTMENT Under CONSTRAints FOR CONTINUOUSLY Monitored RUIN Probabilities}

If instead of only guaranteeing a ruin probability constraint at the time horizon $T$ we are obliged to guarantee this constraint on the whole interval $[0, T]$ then the appropriate constraint to look at is

$$
P\left\{\inf _{t \in[0, T]} Y(\Pi, t) \leq 0\right\} \leq \alpha .
$$

Of course, for a general trading strategy this probability is hard to compute although one might use our computations of the previous sections as a lower bound for the ruin probability as we have

$$
\sup _{t \in[0, T]} P\{Y(\Pi, t) \leq 0\} \leq P\left\{\inf _{t \in[0, T]} Y(\Pi, t) \leq 0\right\} \leq \alpha .
$$

The optimization problem (6), that is when ruin is monitored continuously, can be treated in a similar way to our preceding analysis.

Note that the set of investment strategies that satisfy the constraint in (6),

$$
\{\Pi \in \Theta: P\{Y(\Pi, t) \leq 0\} \leq \alpha \text { for all } t \leq T\},
$$

can be expressed equivalently as

$$
\bigcap_{t \leq T}\{\Pi \in \Theta: P\{Y(\Pi, t) \leq 0\} \leq \alpha\} .
$$

We define the following upper bounds for the ruin probability $\alpha$ for each class of admissible strategies:

$$
\bar{B}_{p}:=\inf _{t \in[0, T]} P\left\{R_{t} \leq z_{0}\right\}
$$




$$
\begin{aligned}
& \bar{B}_{b}:=\inf _{t \in[0, T]} \min \left\{\Phi\left(-\frac{b-\frac{1}{2} \sigma^{2}}{\sigma} \sqrt{t}\right), \Phi\left(\frac{b-\frac{1}{2} \sigma^{2}}{\sigma} \sqrt{t}\right)\right\} \\
& \bar{B}_{a}:=\inf _{t \in[0, T]} \min \left\{\Phi\left(-\frac{b}{\sigma} \sqrt{t}\right), \Phi\left(\frac{b}{\sigma} \sqrt{t}\right)\right\} .
\end{aligned}
$$

Recall that we identify an admissible investment strategy $\Pi \in \Theta$ with its associated fraction, initial capital or amount $\pi \in \mathbb{R}$ and write $Y(\pi, t)=Y(\Pi, t)$.

Theorem 4.1. Let $i \in\{p, b, a\}$. Assume that for a given confidence level

$$
\alpha<\bar{B}_{i}
$$

there exists a strategy $\pi \in \Theta_{i}$ such that

$$
P\{Y(\pi, t) \leq 0\} \leq \alpha \text { for all } t \leq T .
$$

Let $U$ denote the utility function of the insurer. If the expected utility of terminal wealth $E[U\{Y(\pi, T)\}]$ is continuous in $\pi \in \mathbb{R}$ or if $E[U\{Y(\pi, T)\}]$ is monotone increasing in $\pi$, then there exists a finite solution $\pi^{*}$ of optimization problem (6) in the class of investment strategies $\Theta_{i}$. In the latter case, $\pi^{*}$ is given by the largest value for which the constraint is fulfilled.

Proof: Let $i \in\{p, b, a\}$. Lemma 2.1 implies that for $t \geq 0$ and for $\alpha<\bar{B}_{i}$ the set

$$
\{\pi \in \mathbb{R}: P\{Y(\pi, t) \leq 0\} \leq \alpha\}
$$

is compact. Thus, the intersection

$$
\bigcap_{t \leq T}\{\pi \in \mathbb{R}: P\{Y(\pi, t) \leq 0\} \leq \alpha\}
$$

is a compact set, which is non-empty by assumption. Therefore, if $\mathrm{E}[U\{Y(\pi, T)\}]$ is continuous or if $\mathrm{E}[U\{Y(\pi, T)\}]$ is monotone increasing in $\pi$, then an optimal solution $\pi^{*} \in \Theta_{i}$ to optimization problem (6) exists and is finite. If $\mathrm{E}[U\{Y(\pi, T)\}]$ is monotone increasing, then $\pi^{*}$ is given by

$$
\pi^{*}=\sup \left\{\pi \in \Theta: \sup _{t \leq T}\{P[Y(\pi, t) \leq 0] \leq \alpha\}\right\} .
$$

This proves the theorem. 
TABLE 1

OPTIMAL PROPORTION OF WEALTH INVESTED IN RISKY ASSET.

\begin{tabular}{c|c|c|c|c}
\hline \hline & \multicolumn{2}{|c|}{ Pareto } & \multicolumn{2}{c}{ Exp } \\
\cline { 2 - 5 } & $z_{0}=x_{0}$ & $z_{0}=0.1 x_{0}$ & $z_{0}=x_{0}$ & $z_{0}=0.1 x_{0}$ \\
\hline$\alpha=0.05$ & 1.45 & 9 & 0.9 & 6 \\
$\alpha=0.01$ & 1.15 & 8.25 & 0.5 & 3.75 \\
\hline \hline
\end{tabular}

TABLE 2

OPTIMAL AMOUNT INVESTED IN RISKY ASSET AS PROPORTION OF INITIAL CAPITAL $x_{0}$. THE RUIN PROBABILITY IS INDEPENDENT OF $z_{0}$.

\begin{tabular}{l|c|c}
\hline \hline & Pareto & Exp \\
\hline$\alpha=0.05$ & 1.2 & 1 \\
$\alpha=0.01$ & 1.04 & 0.55 \\
\hline \hline
\end{tabular}

\section{NumericAl EXAMPLES AND CONCLUSION}

Although the optimal strategies computed so far are mainly determined as the biggest value such that the ruin constraint is still satisfied, their explicit form in practical situations can be totally different. Even more, these forms call for a careful inspection of the reasons for their appearance. To highlight these aspects, we will consider two cases with nearly identical starting conditions, but with totally different risk profiles: In one example the risk process is dominated by the appearance of many small claims (exponentially distributed case) while in the other it is determined by only a few large claims (Pareto case). For simplicity we assume the interest rate to be zero.

The parameters for the geometric Wiener process are $b=0.05$ and $\sigma=0.2$. The time horizon $T$ is normalized to $T=1$. In the absence of a risk process this would lead to an optimal constant portfolio of $\pi=1.25$ for the choice of the log-utility function. It is therefore interesting to see how the optimal strategy will change in the presence of a risk process and a ruin probability constraint.

The exponential distribution for the claim sizes has parameter $v=2.2585$. Claims arrive according to a homogeneous Poisson process with parameter $\lambda=50$.

The Pareto distribution for the claim sizes has parameter $k=2$. This choice implies that claim sizes have infinite second moment reflecting its purpose as a large claims distribution. Claims arrive according to a homogeneous Poisson process with parameter $\lambda=1$.

The premium rate $c$ is chosen as $c=\mathrm{E}\left[\sum_{i=1}^{N_{1}} X_{i}\right]$. The initial capital $x_{0}$ is chosen so that the given ruin probability $\alpha$ is met when no capital is invested 


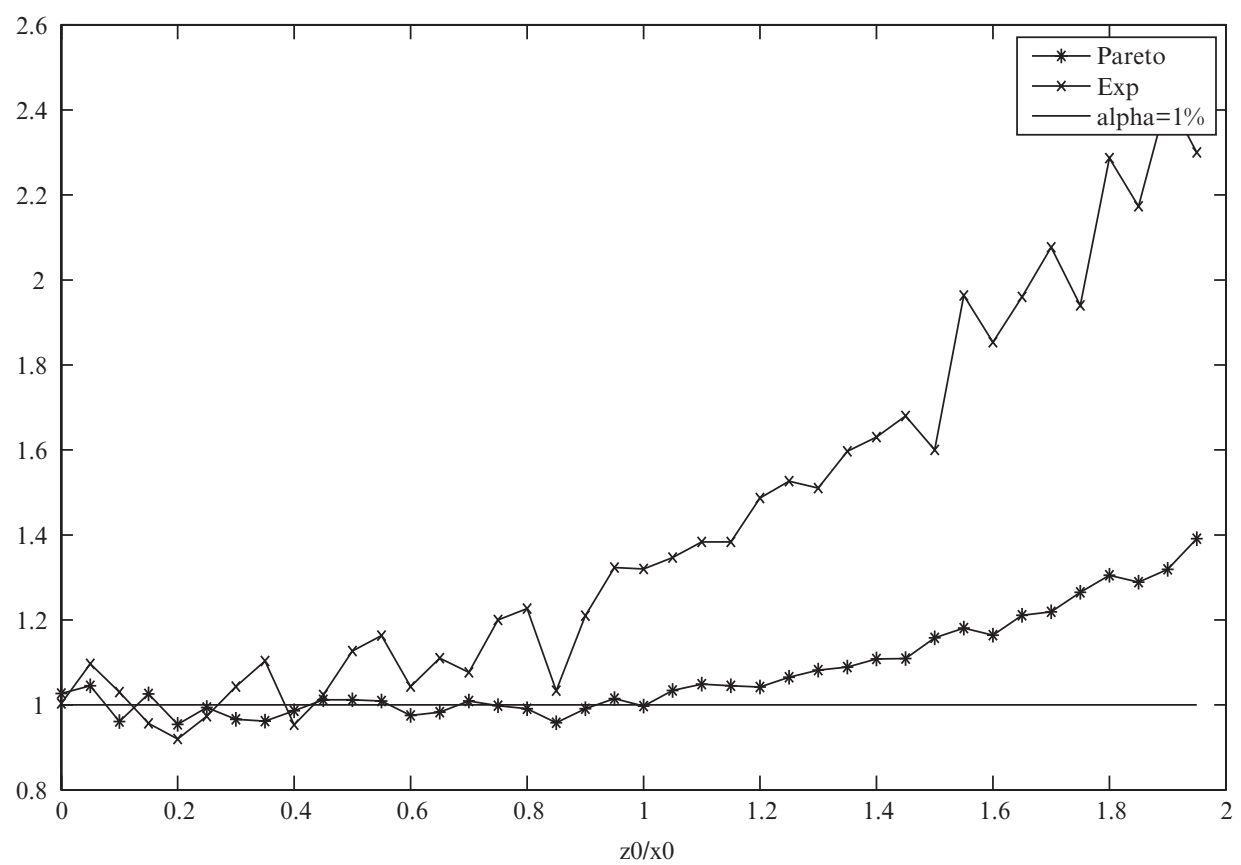

FIGURE 1. Optimal buy-and-hold strategy relative to initial capital for claim sizes with exponential distribution and with Pareto distribution.

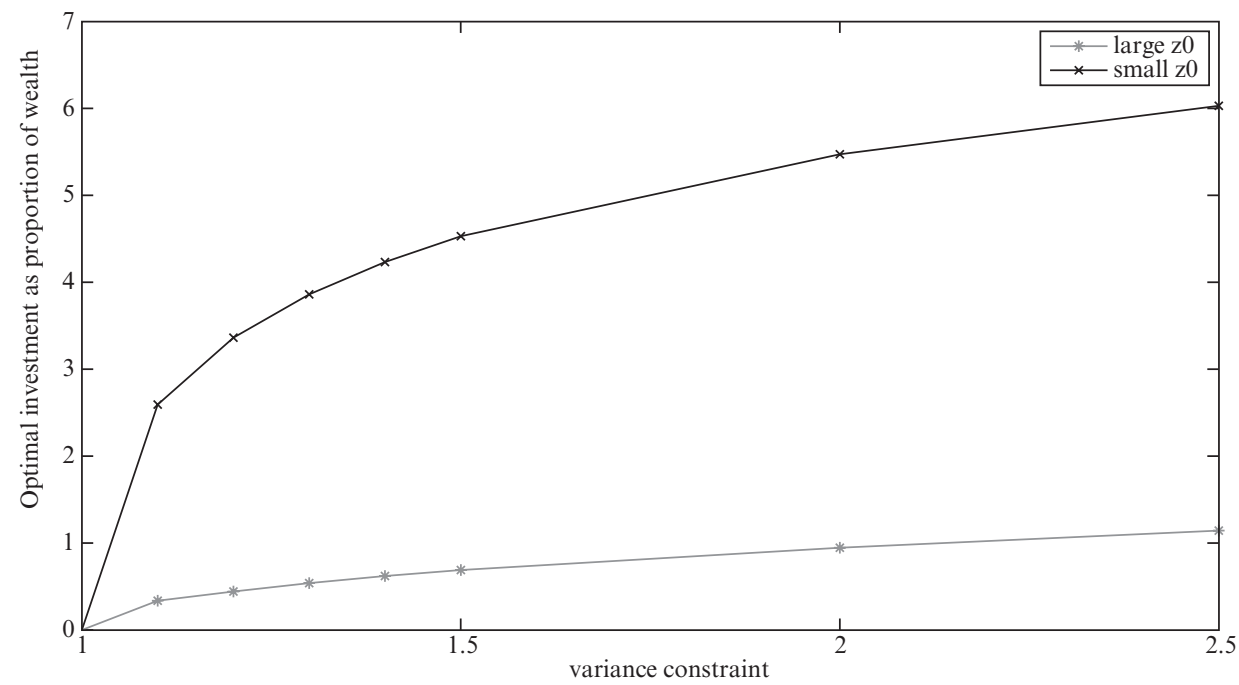

FIGURE 2. Optimal proportion of wealth invested in risky asset under mean-variance criterion for

$$
z_{0}=E\left[\sum_{i=1}^{N_{1}} X_{i}\right] \text { and for } z_{0}=0.1 \times E\left[\sum_{i=1}^{N_{1}} X_{i}\right] \text {. }
$$


in the risky asset. The parameter $v$ of the exponential claims distribution was chosen above so that for $\alpha=0.01$ the initial capital and thus the expected wealth without investment in the risky asset for exponentially distributed claims and for Pareto distributed claims are similar in size, $x_{0}=10.4550$ and $x_{0}=$ 10.4204 , respectively. More importantly, to make our results for both distributions comparable, all strategies considered are given in relative terms either as a proportion of wealth or as a proportion of the initial capital.

We consider an investor who wants to maximize the expected total wealth by investing in the risky asset. The optimal strategy is then given as the largest value for which the ruin constraint is fulfilled.

In Table 1 the optimal investment strategies are displayed when a constant proportion of wealth is invested. We see that the optimal proportion is considerably larger for Pareto distributed claim sizes. For this claim size distribution, the risk associated with rare large claims dominates the effect from trading in the risky asset which means that from the ruin point of view a large investment in the profitable asset can be made. Even more, the potential of the risky investment to generate a lot of capital gains before the first big Pareto claim is used to satisfy the ruin probability constraint. This behaviour is corroborated for the constant amount strategies and the buy-and-hold strategies, see Figure 1 for ruin probabilities when using buy-and-hold strategies. We also see in Table 1 and in Table 2 how the size of the optimal strategies decreases as the given ruin probability $\alpha$ decreases. It should be noted that this behaviour occurs even though the initial capital $x_{0}$ is chosen in dependence of $\alpha$, it is decreasing in $\alpha$.

For exponentially distributed claim sizes and when $\alpha=1 \%$, the optimal proportion $\pi^{*} \approx 40-50 \%$, the optimal amount lies between $50 \%$ and $60 \%$ of the initial capital, while for Pareto distributed claim sizes $\pi^{*} \approx 100 \%$.

It is not surprising that in all cases considered it is optimal to invest a positive amount in the risky asset. This is due to the diversifying impact of the investment in an asset that is independent of the claims process.

The situation is completely different for the mean-variance optimal strategies derived in Section 3. Equation (30) implies that the variance of the total wealth of the insurer is increasing in $\pi^{2}$. A non-zero solution in either class of investment strategies considered only exists, if the constraint $\beta$ is larger than the variance of the ruin process. In Figure 2 we show the mean-variance optimal proportion of wealth invested in the risky asset for different amounts of initial capital and a range of variance constraints. From equation (32), we see that the optimal proportion $\pi^{*} \approx \sqrt{\ln \left(\beta-\operatorname{Var}\left(R_{1}\right)\right)}$, a behaviour that can also be observed in Figure 2. In Table 3, we show the optimal amount to be invested in the risky asset and the optimal buy-and-hold strategy under the mean-variance criterion. Both strategies behave like $\sqrt{\left(\beta-\operatorname{Var}\left(R_{1}\right)\right.}$, see equations (34) and (33).

This different behaviour of the optimal strategies in the mean-variance optimal framework and in the ruin probability framework is totally in line with the results of Emmer et al. (2001). This is in particular clear when one 
TABLE 3

MEAN-VARIANCE OPTIMAL STRATEGIES. THE VALUES IN BRACKETS IN COLUMNS 4 AND 5 SHOW THE OPTIMAL INITIAL VALUE ASSOCIATED WITH THE RISKY INVESTMENT $\pi^{*} \in \Theta_{p}$. IN COLUMN $1, \tilde{\sigma}^{2}=\operatorname{Var}\left(\sum_{i=1}^{N_{1}} X_{i}\right)$.

\begin{tabular}{c|c|c|c|c}
\hline \hline$\beta / \tilde{\sigma}^{2}$ & $\begin{array}{c}\text { constant } \\
\text { amount }\end{array}$ & buy-and-hold & $\begin{array}{c}\text { const. prop. } \\
\left(z_{0}=\mathrm{E}\left(\sum_{i=1}^{N_{1}} X_{i}\right)\right)\end{array}$ & $\begin{array}{c}\text { const. prop. } \\
\left(z_{0}=0.1 \times \mathrm{E}\left(\sum_{i=1}^{N_{1}} X_{i}\right)\right)\end{array}$ \\
\hline 1 & 0 & 0 & 0 & 0 \\
1.1 & 7.000836 & 6.592919 & $0.336407(7.44757)$ & $2.59325156(5.741093)$ \\
1.2 & 9.900677 & 9.323796 & $0.442086(9.787152)$ & $3.36200845(7.443012)$ \\
1.3 & 12.125804 & 11.419271 & $0.538775(11.927724)$ & $3.8615707(8.548972)$ \\
1.4 & 14.001672 & 13.185839 & $0.619396(13.712554)$ & $4.233266486(9.371854)$ \\
1.5 & 15.654345 & 14.742216 & $0.689716(15.269335)$ & $4.529151087(10.026901)$ \\
2 & 22.138588 & 20.848642 & $0.946035(20.943868)$ & $5.473167189(12.116819)$ \\
2.5 & 27.114122 & 25.534267 & $1.142986(25.3041)$ & $6.030489984(13.350653)$ \\
\hline \hline
\end{tabular}

realizes that the constraint on the ruin probability in our model is equivalent to their constraint on the value at risk (or capital at risk).

In total, by restricting to various special (but practically relevant) classes of investment strategies, we have been able to solve the optimal investment problem for an insurer who is also subject to a constraint on the ruin probability. We have considered the case when ruin is only monitored at maturity as well as a permanent ruin probability constraint, which is important under liquidity aspects. An interesting aspect of future research would be the explicit consideration of a bond market, in particular under the assumption that the different factors, bond market, stock market and insurance claims, are correlated. However, as in this paper, computing the ruin probabilities could only be done numerically.

\section{ACKNOWLEDGMENTS}

We thank the anonymous referees for their helpful comments and suggestions.

\section{REFERENCES}

BASAK, S. and SHAPIRO, A. (2001) Value-at-risk-based risk management: optimal policies and asset prices. The Review of Financial Studies 14, 371-405.

BRowne, S. (1995) Optimal investment policies for a firm with a random risk process: exponential utility and minimizing the probability of ruin. Mathematics of Operations Research 20, 937-958.

Emmer, S. and KlÜPPELBerg, C. (2004) Optimal portfolios when stock prices follow an exponential Lévy process. Finance and Stochastics 8, 17-44.

Emmer, S., KlÜPPELberg, C. and Korn, R. (2001) Optimal portfolios with bounded capital at risk. Mathematical Finance 11, 365-384. 
FERSON, W.E. and SCHADT, R.W. (1996) Measuring fund strategy and performance in changing economic conditions. The Journal of Finance 51, 425-461.

Gandy, R. (2005) Portfolio optimization with risk constraints. PhD dissertation, University of Ulm.

Gaier, J., Grandits, P. and Schachermayer, W. (2003) Asymptotic ruin probabilities and optimal investment. Annals of Applied Probability 13, 1054-1076.

HipP, C. and Plum, M. (2000) Optimal investment for insurers. Insurance: Mathematics and Economics 27, 215-228.

HipP, C. and Plum, M. (2003) Optimal investment for investors with state-dependent income, and for insurers. Finance and Stochastics 7, 299-321.

KorN, R. and KorN, E. (2001) Option pricing and portfolio optimization: modern methods of financial mathematics. American Mathematical Society.

LovisceK, A.L. and Jordan, W.J. (2000) Stock selection based on Morningstar's ten-year, fivestar general equity mutual funds. Financial Services Review 9, 145-157.

Merton, R.C. (1971) Optimum consumption and portfolio rules in a continuous-time model. Journal of Economic Theory 3, 373-413.

NG, K.W., YANG, H. and Zhang, L. (2004) Ruin probability under compound Poisson models with random discount factor. Probability in the Engineering and Informational Sciences 18, $55-70$

PAulsen, J. (1998) Ruin theory with compounding assets - a survey. Insurance: Mathematics and Economics 22, 3-16.

PAULSEN, J. (2002) On Cramér-like asymptotics for risk processes with stochastic return on investments. Annals of Applied Probability 12, 1247-1260.

Perold, A.F., Sharpe, W.F. (1988) Dynamic Strategies for Asset Allocation. Financial Analysts Journal Jan/Feb, 16-27.

SCHMidLI, H. (2005) On optimal investment and subexponential claims. Insurance: Mathematics and Economics 36, 25-35.

Yuen, K.C., WANG, G. and NG K.W. (2004) Ruin probabilities for a risk process with stochastic return on investments. Stochastic Processes and their Applications 110, 259-274.

RALF KORN

Department of Mathematics,

University of Kaiserslautern and

Fraunhofer Institute for Industrial Mathematics

Kaiserslautern, 67653 Kaiserslautern

Germany

E-Mail:Korn@mathematik.uni-kl.de

ANKE WIESE

School of Mathematical and Computer Sciences and

the Maxwell Institute for Mathematical Sciences

Heriot-Watt University

Riccarton, Edinburgh EH14 4AS

United Kingdom

E-Mail:A.Wiese@ma.hw.ac.uk 